\title{
Towards a chatbot for digital counselling
}

\author{
Gillian Cameron \\ Ulster University \\ Belfast, N. Ireland \\ g.cameron@ulster.ac.uk
}

Raymond Bond

Ulster University

Belfast, N. Ireland rb.bond@ulster.ac.uk

\author{
David Cameron \\ Inspire Workplaces \\ Belfast, N. Ireland \\ d.cameron@inspirewellbeing.org
}

\author{
Maurice Mulvenna \\ Ulster University \\ Belfast, N. Ireland \\ md.mulvenna@ulster.ac.uk
}

\author{
Gavin Megaw \\ Inspire Workplaces \\ Belfast, N. Ireland \\ g.megaw@inspirewellbeing.org
}

\author{
Cherie Armour \\ Ulster University \\ Belfast, N. Ireland \\ c.armour1@ulster.ac.uk \\ Michael McTear \\ Ulster University \\ Belfast, N. Ireland \\ mf.mctear@ulster.ac.uk
}

\begin{abstract}
The aim of this paper is to outline the design of a chatbot to be used within mental health counselling. One of the main causes of the burden of disease worldwide is mental health problems. Mental health contributes to $28 \%$ of the total burden of disease, compared to $16 \%$ each for cancer and heart disease in the UK. Stress, anxiety or depression accounted for $\mathbf{1 5 . 8}$ million days of sickness absence across the UK in 2016. By 2020, the gap between the demand for mental health care and the resources the National Health Service (NHS) can provide is likely to widen, therefore providers are increasingly needing to find more cost-effective ways to deliver mental health care. Digital Interventions have been created to help with these issues, for example anxiety, stress and depression. Chatbots can be incorporated into digital interventions, or used as standalone interventions. Chatbots can be a more interactive experience for the user to receive information, or complete diagnostic tools, or to even be used for counselling. A demo chatbot was created using interactive emoji's and GIFs to improve the user experience when searching for online self-help tips. This chatbot will be further developed and incorporated into a full web based programme for mental health in the workplace. It is envisaged that the chatbot will be able to provide initial counselling, and lead users into the correct services or self-help information.
\end{abstract}

\section{Mental Health, Counselling, Digital Interventions, Chatbots, Artificial Intelligence, API.ai}

\section{INTRODUCTION}

The aim of this paper is to propose the design of a chatbot to be used in mental health counselling. The first section of this paper gives an overview of the background of digital interventions. The next section gives an overview of chatbots, especially within mental health. A demo chatbot application is discussed with future work proposed.

\section{BACKGROUND}

One of the main causes of the burden of disease worldwide is mental health problems [1]. In the UK, mental health constitutes $28 \%$ of the total burden of disease, compared to $16 \%$ each for cancer and heart disease [2]. Statistics now show that one in four people in the UK will experience a mental health problem in any given year [3]. The Office for
National Statistics reports that in England between October 2014 and September 2015 40\% of people with a mental health problem or learning disability were in employment [4]. Stress, anxiety or depression accounted for 15.8 million days of sickness absence across the UK in 2016 [5]. In the UK, it is estimated that $£ 1,035$ per employee is lost due to mental health annually [6]. Employers are now recognising the cost of this problem and implementing strategies to provide support for employees experiencing mental health issues [7].

\subsection{Digital interventions in mental health}

Digital interventions (DIs) can be defined as any intervention that is accessed by a person on a computer or mobile phone. Dls have been created to help with weight loss [8], smoking [9], anxiety management [10] [11] and other mental health disorders such as depression [12] and Borderline 
Personality Disorder (BDP) [13]. By 2020, the gap between the demand for mental health care and the resources the National Health Service (NHS) can provide is likely to widen, therefore providers are increasingly needing to find more cost-effective ways to deliver mental health care [14]. In his longterm view on health report, Derek Wanless set out a vision that by 2022 people will be taking more responsibility for their own health, through digital resources [15]. Technology is being used by increasing numbers of people, with $87.9 \%$ of adults in the UK (45.9 million) recently using the internet, compared to $86.2 \%$ in 2015 [16].

NHS England is working towards improving waiting times for Cognitive Behavioural Therapy (CBT), however patients could still be subject to substantial delays [17]. One solution is to use technology to computerise CBT, known as Computerised Cognitive Behavioural Therapy (CCBT), which can provide the potential benefits of traditional CBT with fewer face-to-face services [18]. When participants fully engage with CCBT programmes, research has shown that they can be more effective than traditional treatments for depression [19]. Kessler et al., (2009) evaluated the use of an online CBT intervention, however in their program it was not automated, but delivered on an individual basis by an online therapist [20]. Compared with patients receiving traditional GP care, the trial group using the online CBT intervention were more likely to recover.

\section{LITERATURE REVIEW}

\subsection{Overview of Chatbots}

Initially chatbots, or conversational agents, were restricted, simulating very simple conversation between a human and a computer in a scripted way. For example, the very first chatbot created, Eliza, was given a script to be able to maintain a conversation with its human counterpart [21]. Presently, chatbots can complete semantic analysis of the text that the user inputs, to provide a more tailored response [22]. Chatbots are now being used successfully as a means of providing useful information. In one study, a chatbot enabled adolescents to ask questions about sex, drugs and alcohol, and the information provided was found to be useful compared to traditional information outlets or search engines [23]. Educational institutions utilise chatbots, as they provide a more interactive style of e-learning for students [24] [25]. Chatbots can be used to help psychology students improve their skills in diagnosing Generalized Anxiety Disorder (GAD) [26]. Chatbots can benefit sales companies, by providing an enhanced customer service [27]. Responses to frequently asked questions can be automated, freeing up human agents to tackle more difficult queries and problems.

\subsection{Chatbots in healthcare}

Chatbots can be used to help diabetic patients control their diabetes and receive advice [28]. Kumar et al. (2016) proposed a healthcare conversational agent that will allow users to input their health queries [29]. This can facilitate instant replies to users, when they usually are required to wait long periods of time. In response to a user's input that does not match the keywords specified, Kumar et al. have specified that a doctor's contact details will be provided. In their review of artificial intelligence in healthcare, llić and Marković (2016) present the advantages of using conversational agents in healthcare, including cost reduction, improving efficiency and reduction of time spent asking questions to make the right diagnosis [30]. The use of chatbots has also been explored in the pharmaceutical industry. Comendador et al., (2015) introduced a chatbot created with the aim of prescribing, suggesting and giving information on medicine for children [31]. This chatbot uses natural language processing to determine responses to the user's questions.

\subsection{Chatbots in mental health}

Little research has been done on the impact of chatbots on mental health [32], although there has been some discussion of their potential for overcoming certain barriers in mental health, such as waiting lists and geographical problems that hinder attendance at face-to-face counselling appointments [33]. SimCoach is an intelligent virtual human agent that aims to initiate user engagement, increase awareness of symptoms and treatments for users who may be reluctant to talk to a traditional counsellor [34]. Another solution available utilises a web camera and a set of questions to assess a user's level of self-esteem and anxiety. The overall aim is improving a user's wellbeing, reducing anxiety and anger by helping them to cope with stressful situations [35]. Chatbots have been used within the area of student counselling. Kavakli, Li and Rudra (2012) explored the idea of using a chatbot to help students overcome exam stress, as during exam time university counselling services can reach their peak capacity [36]. In their study on students, Bhakta, Savin-Baden, and Tombs (2014) found that they perceived disclosing sensitive information to a chatbot as "safe" [37].

\section{DEVELOPMENT OF A CHATBOT FOR MENTAL HEALTH}

\subsection{Demo Chatbot}


A demo chatbot has been developed to facilitate a more interactive user journey when accessing a self-help library. The self-help library consists of categories such as Anxiety, Depression, Obesity, and Alcohol/Drug misuse. Each category contains numerous PDF files with information on each of the categories. This approach to displaying information is not very interactive for the user, which may deter them from viewing the information at all. The user is required to click into the various categories to find the information they are looking for. The demo chatbot has been created to provide a more interactive way of leading the user into the PDF worksheets, and asking them which areas they would like to receive information on.

\subsubsection{Use of Emoji's}

Emojis (images depicting facial expressions) have been used to convey emotions and moods. For example, Mind (2017) created a mobile application for students, where the sole purpose is to use emoji's. Students can take pictures, and place an emoji on top of the image, to share and track their moods over time [38]. Midkiff and Wyatt (2008) found emoticons or emojis can be used to express the varying degrees of emotions that people may have when using digital health interventions [39]. In the demo chatbot application created, emojis are used to allow the user to select a mood or feeling they are experiencing, such as happy, sad or angry (Figure 1.)

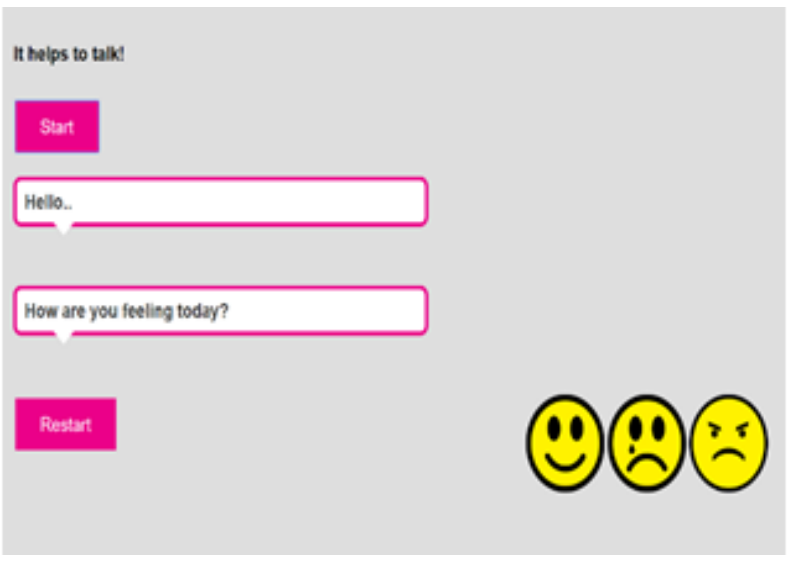

Figure 1: Chatbot first screen

The user can click on their selected emoji, which will then lead into appropriate responses from the chatbot. The user can then pick from several issues they may be having problems with, such as insomnia (Figure 2), bereavement or anxiety for example. The user will then be provided with a brief overview of the issue, and some tips on how to address the issue they may be experiencing.

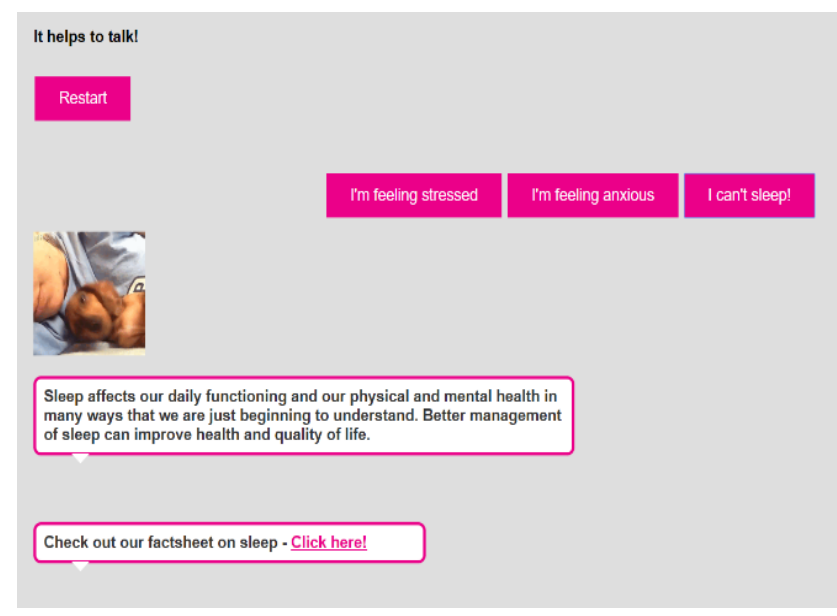

Figure 2: - Chatbot second screen

\subsection{Future work}

\subsubsection{Chatbots as a diagnostic tool}

There is potential to use chatbots to transform diagnostic scales into a more user-friendly way to screen for mental health issues. Currently, most validated screening instruments are usually administered in a paper based format before counselling. Online versions of these screening instruments are available, but can be laborious to fill in for the user. This may potentially deter the user from responding correctly to the questions.

\subsubsection{Proposed conversation flow of the chatbot}

Figure 3 shows a conversation flow for an interactive chatbot conversing with users who are experiencing stress in the workplace. If the user indicates that they are feeling okay, they will be given randomised tips on general wellbeing, and given the option to exit out or restart the chatbot. Another pathway, for general difficulties that are not related to the workplace, will lead into a wide variety of issues that the user can choose from to receive more information and self-help tips. Another pathway will lead into a general stress programme, which has been devised by a clinical psychologist. A specific pathway has been created for users who are experiencing negative feelings directly from reviewing disturbing online content, leading into a screening tool based on the impacts of events scale for the assessment of trauma. This chatbot will be incorporated into a wider web application that will screen and provide support to employees who are working with disturbing online media in the workplace. 


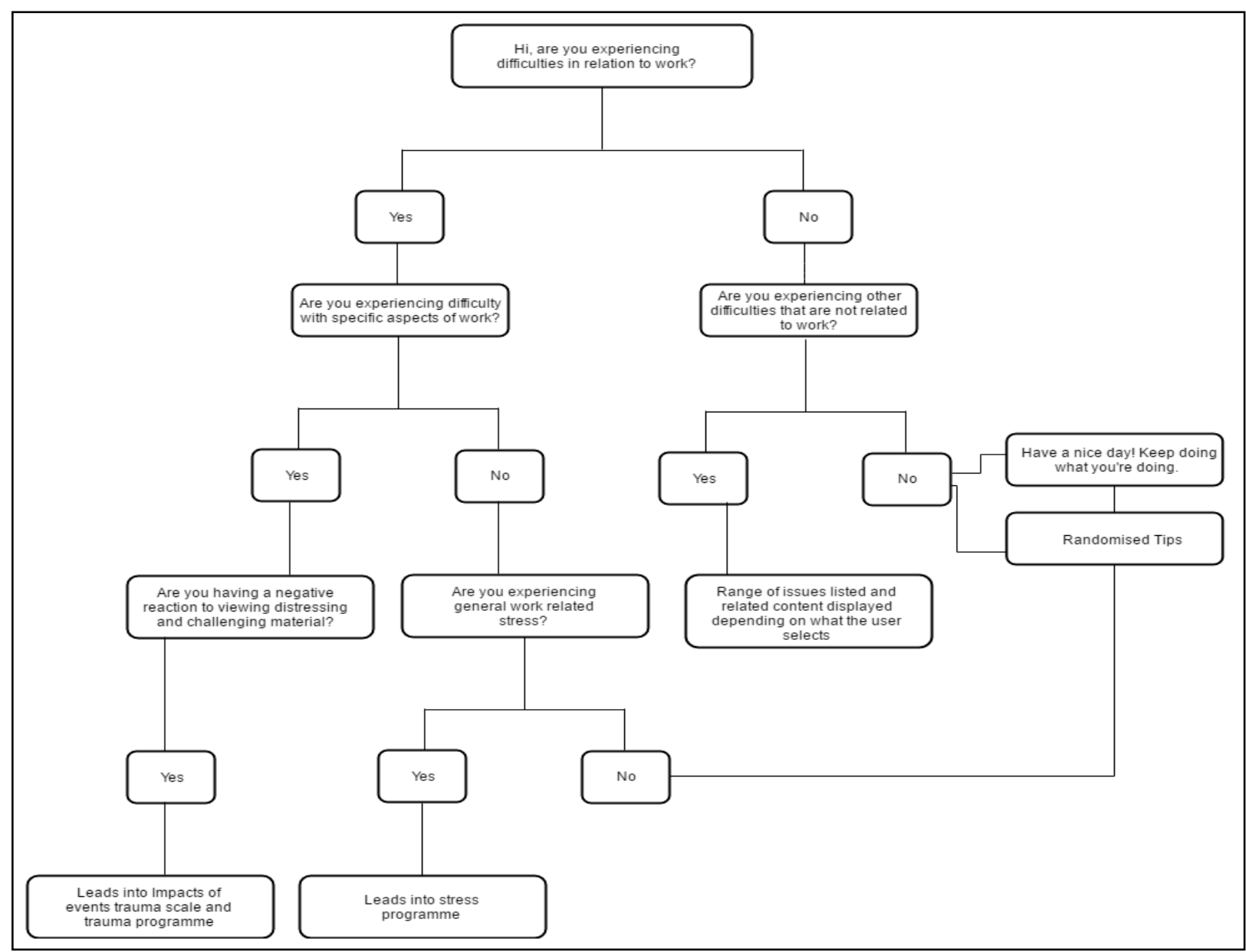

Figure 3: Proposed Chatbot script

\subsubsection{Artificial Intelligence}

Facebook's Messenger application is already installed on most people's smart phones, and has over 1.2 billion monthly active users [40]. Chatbots can be used on existing platforms such as Messenger, making it easier for the user to engage with a business or service. API.ai is a popular natural language understanding platform that allows developers to create conversational user interfaces and incorporate them into their own applications. API.ai will be used to allow the user to have more interaction with the chatbot, as the current demo is limited. The demo can only provide the user with a scripted interaction, but platforms such as API.ai will be utilised to allow the user to input free text, and establish a more flexible conversational interface. This platform can be programmed to recognise the natural language inputted by a user, and can recognise their intent to provide more coherent responses. The proposed platform architecture will consist of a bot engine (API.ai), a web application, and a chat platform such as Facebook Messenger or Skype.

\subsubsection{Ethical considerations}

The demo chatbot currently provides rigid responses and has no option for the user to type freely. Once the final chatbot has been developed, a study on measuring the empathy of chatbot replies would be considered, to improve on any areas where the chatbot is providing incorrect or apathetic responses. Shawar and Atwell (2007) found it was best to adapt the evaluation to the needs of the user, rather than using established standards such as the Loebner Prize evaluation methodology [41].

Chatbots have the risk of being used inappropriately. In 2016 Microsoft launched a chatbot on twitter, known as Tay. This chatbot was programmed to learn and form replies based on its interactions on twitter. This caused people to interact with the bot inappropriately, using offensive language and content which ultimately caused Microsoft to take the chatbot down after only 16 hours [42].

When developing any digital intervention, great care and attention must be placed on privacy and security [43]. Many studies reiterate this point, as users are less likely to use an intervention if they think their data is not secure enough [44].

Whitby (2014) discusses the lack of responsibility of care or undesirable outcomes for patients using digital interventions in healthcare. Furthermore, they question if deceiving a patient into thinking that they are interacting with a human rather than a 
computer is unethical. They call for the development of a code of conduct, to enable designers to consider these ethical issues when developing [45]. Shevat (2017) discusses the term onboarding, a way for the chatbot to be introduced and to explain its capabilities, this can be utilised to set the expectations of the user [46].

The Wizard of $\mathrm{Oz}$ technique is where a human takes on the role as a computer in a simulated interaction [47]. Using the Wizard of Oz technique could allow the design and conversation flow to be tested during the design phase.

The chatbot will need to be designed to meet the needs of the user, by providing empathetic and relevant responses. The chatbot will require appropriate responses to unknown requests from the user to minimise incorrect responses being sent.

\section{CONCLUSION}

Using a chatbot for mental health counselling can provide many benefits for the user. Chatbots can give a user instant information. By incorporating mental health screening tools into a chatbot interface, the user can have a more interactive and user-friendly experience. Research has shown users find chatbots "safe" and easy to talk to. Chatbots can create another option for users who do not want to receive face to face treatment, however there are many ethical aspects to consider.

\section{ACKNOWLEDGEMENTS}

The research undertaken for this paper has been supported by UK Knowledge Transfer Partnership under KTP grant ID 1022267.

\section{REFERENCES}

[1] Vos, T., Barber, R.M., Bell, B., Bertozzi-Villa, A., Biryukov, S., Bolliger, I., Charlson, F., Davis, A., Degenhardt, L. and Dicker, D. 2015. 'Global, regional, and national incidence, prevalence, and years lived with disability for 301 acute and chronic diseases and injuries in 188 countries, 1990-2013: a systematic analysis for the Global Burden of Disease Study 2013', The Lancet, 386(9995), pp. 743

[2] Ferrari, A., Charlson, F., Norman, R., Patten, S., Freedman, G., Murray, C., Vos, T. and Whiteford, H. 2013. Burden of Depressive Disorders by Country, Sex, Age, and Year: Findings from the Global Burden of Disease Study 2010. PLoS Med, 10(11), p.e1001547
[3] McManus S, Meltzer H, Brugha T, Bebbington $P$, Jenkins $R$ (eds), 2009. Adult psychiatric morbidity in England, 2007: Results of a household survey. [online] NHS Information Centre for Health and Social Care, pp.1-274. Available at: http://www.hscic.gov.uk/catalogue/PUB02931/ad ulpsyc-morbres-hou-sur-eng-2007-rep.pdf [Accessed 03 Mar. 2017].

[4] Office for National Statistics. 2016. NOMIS Database, Annual population survey analysis Variables (percentages) [Accessed 03 Feb. 2017].

[5] Office for National Statistics. 2017. Sickness absence in the labour market: 2016. [online] Available

at: https://www.ons.gov.uk/employmentandlabourm arket/peopleinwork/labourproductivity/articles/sic knessabsenceinthelabourmarket/2016 [Accessed 4 Feb. 2017].

[6] ERS Research and Consultancy, 2016. Health at Work: Economic Evidence Report 2016. [online] Available at: https://www.bhf.org.uk//media/files/healthatwork/health_at_work_economic_evidence_rep ort_2016.pdf. [Accessed 7 Jan. 2017].

[7] Hudson, M. 2016. The Management of Mental Health at Work. [online] Available at: http://www.acas.org.uk/media/pdf/r/2/Mental_he alth_r eport_11_Nov_2016.pdf [Accessed 6 Mar. 2017].

[8] Gold, B.C., Burke, S., Pintauro, S., Buzzell, P. and Harvey-Berino, J., 2007. Weight loss on the web: A pilot study comparing a structured behavioural intervention to a commercial program. Obesity, 15(1), pp. 155-155.

[9] Munoz, R.F., Lenert, L.L., Delucchi, K., Stoddard, J., Perez, J.E., Penilla, C. and PerezStable, E.J. 2006. 'Toward evidence-based Internet interventions: A Spanish/English Web site for international smoking cessation trials', Nicotine \& tobacco research: official journal of the Society for Research on Nicotine and Tobacco, 8(1), pp. 77-87.

[10] Frazier, P., Richards, D., Mooney, J., Hofmann, S.G., Beidel, D., Palmieri, P.A. and Bonner, C., 2016. Acceptability and proof of concept of internet-delivered treatment for depression, anxiety, and stress in university students: protocol for an open feasibility trial. Pilot and Feasibility Studies, 2(1), pp. 28.

[11] Christensen, H., Griffiths, K.M. and Korten, A., 2002. Web-based cognitive behaviour therapy: analysis of site usage and changes in depression and anxiety scores. Journal of Medical Internet Research, 4(1), pp. e3. 
[12] Clarke, G., Eubanks, D., Reid, E., Kelleher, C., O'Connor, E., Debar, L.L., Lynch F., Nunley, S. and Gullion, C., 2005. Overcoming Depression on the Internet (ODIN)(2): a randomized trial of a self-help depression skills program with reminders. Journal of medical Internet research, 7(2), pp. e16.

[13] Rizvi, S.L., Dimeff, L.A., Skutch, J., Carroll, D. and Linehan, M.M., 2011. A pilot study of the DBT coach: an interactive mobile phone application for individuals with borderline personality disorder and substance use disorder. Behavior therapy, 42(4), pp. 589-600.

[14] Hollis, C., Morriss, R., Martin, J., Amani, S., Cotton, R., Denis, M. and Lewis, S., 2015. Technological innovations in mental healthcare: harnessing the digital revolution. The British journal of psychiatry: the journal of mental science, 206(4), pp. 263-265.

[15] Wanless, D. 2002. 'Securing our future health: taking a long-term view', London: HM Treasury, 16.

[16] Office for National Statistics. 2016. Internet users in the UK- Office for National Statistics. [online] Available at: https://www.ons.gov.uk/businessindustryandtrad e/itandinternetindustry/bulletins/internetusers/20 16 [Accessed 5 Feb. 2017].

[17] Mind, 2013. We still need to talk A report on access to talking therapies. [online] London. Available at: http://www.mind.org.uk/media/494424/we-stillneedtotalk_report.pdf [Accessed 6 Mar. 2017].

[18] Kaltenthaler, E., Brazier, J., De Nigris, E., Tumur, I., Ferriter, M., Beverley, C., Parry, G., Rooney, G. and Sutcliffe, P. 2006. 'Computerised cognitive behaviour therapy for depression and anxiety update: a systematic review and economic evaluation', Health technology assessment, 10(33), pp. 1-186.

[19] Kaltenthaler, E., Parry, G., Beverley, C. and Ferriter, M. 2008. 'Computerised cognitivebehavioural therapy for depression: systematic review', The British journal of psychiatry: the journal of mental science, 193(3), pp. 181- 184. doi: 10.1192/bjp.bp.106.025981 [doi].

[20] Kessler, D., Lewis, G., Kaur, S., Wiles, N., King, M., Weich, S., Sharp, D.J., Araya, R., Hollinghurst, S. and Peters, T.J. 2009. 'Therapist-delivered Internet psychotherapy for depression in primary care: a randomised controlled trial', The Lancet, 374(9690), pp. 628634.

[21] Weizenbaum, J. 1975. Computer power and human reason. 1st ed. San Francisco: W.H. Freeman, p.3.
[22] Pasikowska, A., Zaraki, A. and Lazzeri, N. 2013. A dialogue with a virtual imaginary interlocutor as a form of a psychological support for well-being. ACM, pp. 16.

[23] Crutzen, R., Peters, G.Y., Portugal, S.D., Fisser, E.M. and Grolleman, J.J. 2011. 'An artificially intelligent chat agent that answers adolescents' questions related to sex, drugs, and alcohol: an exploratory study', Journal of Adolescent Health, 48(5), pp. 514-519.

[24] Kowalski, S., Hoffmann, R., Jain, R. and Mumtaz, M. 2011. 'Using conversational agents to help teach information security risk analysis'.

[25] Bii, P. 2013. 'Chatbot technology: A possible means of unlocking student potential to learn how to learn', Educational Research, 4(2), pp. 218-221.

[26] Morales-Rodríguez, M., González B, J., Florencia Juárez, R., Fraire Huacuja, $H$. and Martínez Flores, J. $2010 . \quad$ 'Emotional conversational agents in clinical psychology and psychiatry', Advances in Artificial Intelligence, , pp. 458-466.

[27] Radziwill, N.M. and Benton, M.C. 2017. 'Evaluating Quality of Chatbots and Intelligent Conversational Agents', arXiv preprint arXiv:1704.04579, .

[28] Lokman, A.S., Zain, J.M., Komputer, F.S. and Perisian, K. 2009. Designing a Chatbot for diabetic patients.

[29] Kumar, V.M., Keerthana, A., Madhumitha, M., Valliammai, S. and Vinithasri, V.2016. 'Sanative Chatbot For Health Seekers'.

[30] Ilić, Dejan T., Marković, B. 2016. 'Possibilities, Limitations and economic aspects of artificial intelligence applications in healthcare', Ecoforum Journal, 5(1).

[31] Comendador, B.E.V., Francisco, B.M.B., Medenilla, J.S. and Mae, S. 2015. 'Pharmabot: a pediatric generic medicine consultant chatbot', Journal of Automation and Control Engineering Vol, 3(2).

[32] Mohr, D.C., Burns, M.N., Schueller, S.M., Clarke, G. and Klinkman, M. 2013. 'Behavioral intervention technologies: evidence review and recommendations for future research in mental health', General hospital psychiatry, 35(4), pp. 332-338.

[33] Miner, A., Chow, A., Adler, S., Zaitsev, I., Tero, P., Darcy, A. and Paepcke, A. 2016. Conversational Agents and Mental Health: Theory-Informed Assessment of Language and Affect. ACM, pp. 123.

[34] Rizzo, A.A., Lange, B., Buckwalter, J.G., Forbell, E., Kim, J., Sagae, K., Williams, J., 
Rothbaum, B.O., Difede, J. and Reger, G. 2011. An intelligent virtual human system for providing healthcare information and support.

[35] Pasikowska, A., Zaraki, A. and Lazzeri, N. 2013. A dialogue with a virtual imaginary interlocutor as a form of a psychological support for well-being. ACM, pp. 16.

[36] Kavakli, M., Li, M. and Rudra, T. 2012. 'Towards the development of a virtual counselor to tackle students' exam stress', Journal of Integrated Design and Process Science, 16(1), pp. 5-26.

[37] Bhakta, R., Savin-Baden, M. and Tombs, G. 2014. Sharing Secrets with Robots? pp. 2295.

[38] Mind, 2017. Are emojis the future of mental health?. [online] Available at: https://mind.org.uk/informationsupport/yourstories/are-emojis-the-future-of-mentalhealth [Accessed 1 May 2017].

[39] Midkiff, D.M. and Joseph Wyatt, W. 2008. 'Ethical issues in the provision of online mental health services (etherapy)', Journal of Technology in Human Services, 26(2-4), pp. 310-332.

[40] Statista. 2017. Facebook Messenger: monthly active users 2017 | Statistic. [online] Available at: https://www.statista.com/statistics/417295/faceb ookmessenger-monthly-active-users/ [Accessed 30 Apr. 2017].

[41] Shawar, B.A. and Atwell, E. 2007. Different measurements metrics to evaluate a chatbot system. Association for Computational Linguistics, pp. 89.

[42] Neff, G. and Nagy, P. (2016) 'Automation, Algorithms, and Politics| Talking to Bots: Symbiotic Agency and the Case of Tay', International Journal of Communication, 10, pp. 17.

[43] Kumar, S., Nilsen, W.J., Abernethy, A., Atienza, A., Patrick, K., Pavel, M., Riley, W.T., Shar, A., Spring, B. and Spruijt-Metz, D., 2013. Mobile health technology evaluation: the mHealth evidence workshop. American Journal of Preventive Medicine, 45(2), pp. 228-236.

[44] Proudfoot, J.G., Parker, G.B., Pavlovic, D.H., Manicavasagar, V., Adler, E. and Whitton, A.E., 2010. Community attitudes to the appropriation of mobile phones for monitoring and managing depression, anxiety, and stress. Journal of Medical Internet Research, 12(5), pp. e64.

[45] Whitby, B. 2014. 'The Ethical Implications of Non-human Agency in Health Care', MEMCA14. This Proceedings.
[46] Shevat, A. 2017. Designing Bots. Creating Conversational Experiences. 1st edn. O'Reilly Media.

[47] Fraser, N.M. and Gilbert, G.N. 1991. 'Simulating speech systems', Computer Speech \& Language, 5(1), pp. 81-99. 